\title{
An Effective Perturbation Iteration Algorithm for Solving Riccati Differential Equations
}

\author{
M. Khalid \\ Department of Mathematical Sciences \\ Federal Urdu University of Arts, Sciences \& Techonology \\ University Road, Karachi-75300, Pakistan \\ Faheem Zaidi \\ Department of Mathematical Sciences \\ Federal Urdu University of Arts, Sciences \& Technology \\ University Road, Karachi-75300, Pakistan
}

\author{
Mariam Sultana \\ Department of Mathematical Sciences \\ Federal Urdu University of Arts, Sciences \& Technology \\ University Road, Karachi-75300, Pakistan \\ Uroosa Arshad \\ Department of Mathematical Sciences \\ Federal Urdu University of Arts, Sciences \& Technology \\ University Road, Karachi-75300, Pakistan
}

\begin{abstract}
In the following study, a novel approach called the Perturbation Iteration Algorithm $P I A$ has been proposed and subsequently adopted for deriving and solving the Riccati differential equation. This new Perturbation Iteration Method is efficient and has no requirement of a small parameter assumption as its earlier classical counterparts do. Some examples have been presented to exhibit how simply and efficiently the proposed method works. After deriving the exact solution of the Riccati equation, the capability and the simplicity of the proposed technique is clarified. A percentage error for each example has also been presented.
\end{abstract}

\section{Keywords:}

Perturbation Iteration Algorithm, Riccati Differential Equation, Analytical Approximation, Convergence

\section{INTRODUCTION}

The Riccati Differential Equations ( $R D E s)$ of the following form:

$$
u^{\prime}(x)=A(x)+B(x) u(x)+C(x) u^{2}(x) \quad x_{\circ} \leq x \leq X
$$

Where $A(x), B(x)$ and $C(x)$ are given functions and $u\left(x_{\circ}\right)=c$, where $c$ is an arbitrary constant. These are a collection of nonlinear differential equations of immense importance, and have a major role to play in various fields of applied sciences [1]. As an example, take the solitary wave solutions of a nonlinear partial differential equation; it can be represented as a polynomial in two basic functions that maintain the criteria of a projective Riccati equation [2]. Problems such as these also crop up in optimal control literature. In spite of that, deriving an analytical solution in a form that is explicit appears unachievable except in very particular situations [3]. Certainly, if the persons concerned have apt knowledge with respect to a particular solution, the derivation of a general solution is fairly easy. For most cases, one must make use of numerical techniques or approximate approaches for the obtainment of solutions. This is why the problem has attracted quite a bit of attention in the recent past and has been the topic of study of many authors [4, 5, 6] and the references cited therein. Attaining analytical derivations for the Riccati equation in an explicit form seems an unlikely concept except for certain special situations as discussed above, however, that can easily be overcome if its one particular solution is known. One has to, then, adopt numerical techniques or approximate approaches for getting its solution. In [6], El-Tawil's study Adomian's Decomposition Method has been put forward for solving the Riccati differential equations. Abbasbandy solved the equation making use of He's $V I M$, Homotopy Perturbation Method and iterating it. He then matched the accuracy of the obtained solution with the result derived through the Adomian Decomposition Method [5, 7, 8]. On top of that, the Homotopy Analysis Method and a piecewise Variational Iteration Method are introduced for the solution of Riccati differential equations [4, 9]. The Taylor, Chebyshev and Legendre (matrix and collocation) methods have been employed by Sezer et al.[10], and Akyuz [11] to solve the famous Riccati equations.

Every listed method has its own limitations and set-backs in its application. For example, the Adomian Decomposition Method and Variation Iteration Method are limited in that the former involves the usage of complicated algorithms in calculating Adomian polynomials for nonlinear problems, and the latter is inherently inaccuracy when it comes to identifying the Lagrange multiplier which is a necessary factor for the construction of a Variational Iteration formula. The Homotopy Perturbation Method requires solving a linear functional equation in each iteration, and this at times proves to be immensely tricky; sometimes even near impossible. The efficiency of Homotopy Analysis Method depends heavily on choosing auxiliary parameter.

Perturbation methods are classic methods that have been around for over a century, and they have proved to be victorious in obtaining approximate analytical solutions. This method has been employed successfully to differential equations, integro differential equations, and algebraic equations. Various perturbation methods, 
such as the method of averaging, the renormalization method, the method of multiple scales, the Lindstedt-Poincare method, the method of matched asymptotic expansions, plus their variants were developed to make our results as accurate as possible [12, 13].

The main drawback of perturbation methods is the prerequisite of a small parameter, which is hard to fulfill. That is why sometimes a small parameter may have to be artificially introduced into the equation. The solutions therefore are not entirely valid. They may be for weakly nonlinear problems, but the validity mostly does not cover strongly nonlinear problems.

Numerous methods have been introduced by researchers in their recent literature to attain admissible solutions which eschew the requirement of a small parameter. Lately, a class of alternative perturbation-iteration algorithms has been proposed, the fundamentals of which were outlined for the first-order differential equations by Pakdemirli et al.[14]. Multiple iteration algorithms can be equated by taking various number of terms in the perturbation expansions and different order of correction terms in the Taylor series expansions. The perturbation iteration algorithm is represented by $\operatorname{PIA}(n, m)$ where $n$ embodies the correction terms in the perturbation expansion and $m$ is the highest order derivative term in the Taylor series. One of the best advantages of the new method is that it does not require initial transformation of the equations to another form. In fact, the technique was first developed for algebraic equations and then tweaked to adapt to ordinary differential equations [14, 15, 16].

In this paper, the iteration algorithm is aptly and tactfully employed to obtain the approximate solution of some nonlinear Riccati differential equations. The competence and accuracy of the method presented is demonstrated with the help of three examples, inclusive of the quadratic Riccati Equation. As it will eventually be proved, the interface of this method is simple and its results fairly accurate Here on wards, the introduction of the Perturbation Iteration Algorithm is presented. In section 3, this algorithm is applied to the Riccati differential equation to solve it with great precision. Conclusive remarks close the paper in Section 4.

\section{METHODOLOGY}

In this section, a perturbation iteration algorithm is developed by taking one correction term in the perturbation expansion and correction terms of only first derivatives in the Taylor Series expansion, i.e. $n=1, m=1$. The algorithm is called $P I A(1,1)$. Consider the general first order differential equation

$$
G(u, \dot{u}, \epsilon)=0
$$

with $u=u(x)$ and $\epsilon$ the perturbation parameter. Only one correction term is taken in the perturbation expansion

$$
u_{1}=u_{\circ}+\epsilon u_{c}+\ldots \ldots
$$

Upon substitution of Eq. 3 into Eq. 2 and expanding in a Taylor series with first derivatives only yields

$$
\begin{aligned}
& G\left(u_{\circ}, \dot{u}_{\circ}, 0\right)+G_{u}\left(u_{\circ}, \dot{u}_{\circ}, 0\right) \epsilon u_{c}+ \\
& G_{\dot{u}}\left(u_{\circ}, \dot{u}_{\circ}, 0\right) \epsilon \dot{u}_{c}+G_{\epsilon}\left(u_{\circ}, \dot{u}_{\circ}, 0\right) \epsilon=0
\end{aligned}
$$

where subscripts denote differentiation with respect to the variable. Reorganizing the equation

$$
\dot{u}_{c}+\frac{G_{u}}{G_{\dot{u}}} u_{c}=\frac{G_{\epsilon}+\frac{G}{\epsilon}}{G_{\dot{u}}}
$$

All derivatives are evaluated at $\epsilon=0$. it is readily observed that the above equation is a variable coefficient first order differential equation whose solution is

$$
\begin{aligned}
u_{c}= & c \exp \left[-\int \frac{G_{u}}{G_{\dot{u}}} d t\right]-\left[\int \frac{G_{\epsilon}+\frac{G}{\epsilon}}{G_{\dot{u}}} \exp \left[\int \frac{G_{u}}{G_{\dot{u}}} d t\right] d t\right] \times \\
& \exp \left[\int \frac{G_{u}}{G_{\dot{u}}} d t\right]
\end{aligned}
$$

Substitution of Eq. 6 into 3 and constructing the iteration scheme yields

$$
u_{n+1}=u_{n}+\epsilon c_{n} u_{c}
$$

In this paper, we only consider a case $n=m=1$ for the sake of simplicity as more Algebra is involved in constructing iteration solutions for $P I A(1,2)$ and $P I A(1,3)$ as compared to $P I A(1,1)$. The percentage error, which we will calculate in next section, is defined as

$\%$ Error $=\left|\frac{\text { Exact Solution }- \text { Numerical Solution }}{\text { Exact Solution }}\right| \times 100$

\section{ILLUSTRATIVE EXAMPLES}

In this work, we use the Perturbation Iteration Algorithm to obtain the approximate solution of some nonlinear Riccati differential equations. Effectiveness and precision of the presented method is shown by three examples.

\subsection{Example: 1}

Consider the following Riccati equation

$$
u^{\prime}(x)=1+2 u(x)-u^{2}(x)
$$

with initial condition $u(0)=0$. For the above differential equation, the exact solution is previously known to be

$$
u(x)=1+\sqrt{2} \tanh \left[\sqrt{2} x+\frac{1}{2} \log \left(\frac{\sqrt{2}-1}{\sqrt{2}+1}\right)\right]
$$

When we apply Perturbation Iteration Algorithm , Eq. 5 implies

$$
\dot{u}^{c}=\frac{-\dot{u}+1}{\epsilon}+2 u-u^{2}
$$

Selecting $u_{\circ}=0$, which satisfies initial condition and using the algorithm of perturbation Iteration method, the approximate solution at each step are

$$
u_{11}=x
$$

$$
u_{12}=x+x^{2}-\frac{1}{3} x^{3}
$$




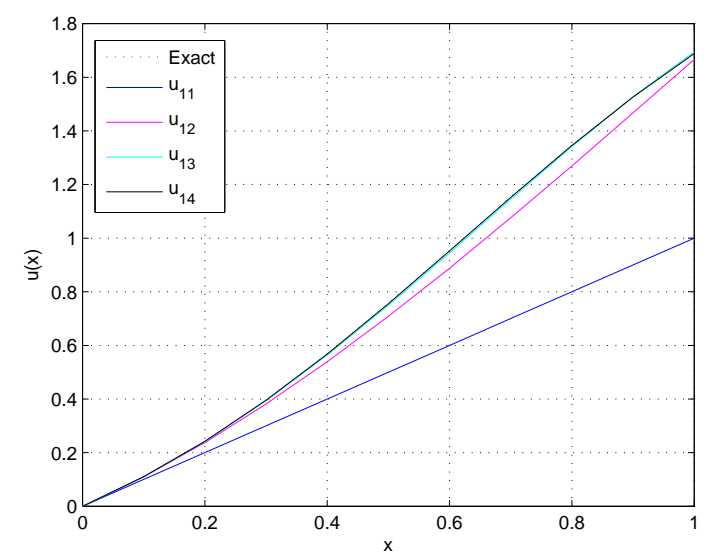

Fig. 1. Comparison of solution obtained by $P I A(1,1)$ with exact solution of Example 1

Table 1. Percentage Error Estimation of Example 1

\begin{tabular}{ccccc}
\hline & \%Errorin & \%Errorin & $\%$ Errorin & $\%$ Errorin \\
$x$ & $u_{11}$ & $u_{12}$ & $u_{13}$ & $u_{14}$ \\
\hline 0.10 & 9.33422050 & 9.66666670 & 0.54660440 & 0.02539510 \\
0.20 & 17.34744810 & 18.66666670 & 1.79167470 & 0.15083750 \\
0.30 & 24.07078750 & 27.00000000 & 3.28491560 & 0.36640910 \\
0.40 & 29.55416880 & 34.66666670 & 4.70540310 & 0.60012770 \\
0.50 & 33.86369300 & 41.66666670 & 5.81582630 & 0.76422920 \\
0.60 & 37.07830780 & 48.00000000 & 6.43644790 & 0.78643160 \\
0.70 & 39.28612450 & 53.66666670 & 6.42952280 & 0.63129470 \\
0.80 & 40.58068960 & 58.66666670 & 5.68957180 & 0.31582940 \\
0.90 & 41.05748040 & 63.00000000 & 4.13708150 & 0.07759120 \\
1.00 & 40.81083440 & 66.66666670 & 1.71428570 & 0.38854650 \\
\hline$\%$ & & & & \\
Mean & 31.29837550 & 42.16666670 & 4.05513340 & 0.41066920 \\
Error & & & & \\
\hline
\end{tabular}

$$
u_{13}=x+x^{2}+\frac{1}{3} x^{3}-\frac{2}{3} x^{4}-\frac{1}{15} x^{5}+\frac{1}{9} x^{6}-\frac{1}{63} x^{7}
$$

$$
\begin{aligned}
u_{14}= & x+x^{2}+\frac{1}{3} x^{3}-\frac{1}{3} x^{4}-\frac{3}{5} x^{5}+\frac{4}{45} x^{6}+\frac{71}{315} x^{7}+ \\
& \frac{17}{420} x^{8}-\frac{62}{945} x^{9}-\frac{62}{4725} x^{10}+\frac{27}{1925} x^{11}-\frac{1}{1890} x^{12}- \\
& \frac{41}{36855} x^{13}+\frac{1}{3969} x^{14}-\frac{1}{59535} x^{15}
\end{aligned}
$$

Figure 1 shows comparison between exact solution and iterative solutions of Example 1.It is clear that accurate value is attained as number of iteration increases. Table 1 illustrate percentage errors of each successive solution of Riccati differential equation by perturbation iteration algorithm.

\subsection{Example: 2}

Consider the following Riccati equation

$$
u^{\prime}(x)=1+x^{2}-u^{2}(x)
$$

with initial condition $u(0)=1$. For the above differential equation, the exact solution is previously known to be

$$
u(x)=x+\frac{e^{-x^{2}}}{1+\int_{0}^{x} e^{-t^{2}} d t}
$$

When we apply Perturbation Iteration Algorithm , Eq.5implies

$$
\dot{u}^{c}=\frac{-\dot{u}+1+x^{2}}{\epsilon}-u^{2}
$$

Selecting $u_{\circ}=1$ and using algorithm of perturbation Iteration Method, the successive solution are

$$
u_{11}=1+\frac{1}{3} x^{3}
$$

$$
\begin{gathered}
u_{12}=1+\frac{1}{3} x^{3}-\frac{1}{6} x^{4}-\frac{1}{63} x^{7} \\
u_{13}=1+\frac{1}{3} x^{3}-\frac{1}{6} x^{4}+\frac{1}{15} x^{5}-\frac{1}{63} x^{7}+\frac{1}{56} x^{8}-\frac{1}{324} x^{9}+ \\
\frac{2}{2079} x^{11}-\frac{1}{2268} x^{12}-\frac{1}{59535} x^{15}
\end{gathered}
$$

$$
\begin{aligned}
u_{14}= & 1+\frac{1}{3} x^{3}-\frac{1}{6} x^{4}+\frac{1}{15} x^{5}-\frac{1}{45} x^{6}-\frac{1}{63} x^{7}+\frac{1}{56} x^{8}- \\
& \frac{34}{28354} x^{9}+\frac{23}{8100} x^{10}+\frac{29}{51975} x^{11}-\frac{53}{33264} x^{12}+ \\
& \frac{107}{126360} x^{13}-\frac{29}{119070} x^{14}-\frac{631}{19646550} x^{15}+ \\
& \frac{1061}{13970880} x^{16}-\frac{5119}{125737920} x^{17}+\frac{23}{2449440} x^{18}+ \\
& \frac{9113}{5375296080} x^{19}-\frac{53}{19646550} x^{20}+\frac{11281}{9901861200} x^{21}- \\
& \frac{1}{8083152} x^{22}-\frac{662}{10438212015} x^{23}+\frac{239}{3960744480} x^{24}- \\
& \frac{23}{1928934000} x^{25}+\frac{4}{3341878155} x^{27}-\frac{1}{1890355320} x^{28}- \\
& \frac{1}{109876902975} x^{31}
\end{aligned}
$$

Figure 2 be evidence for difference between exact and approximate solution obtained

\subsection{Example: 3}

Consider the following Riccati equation

$$
u^{\prime}(x)=1+u^{2}(x)
$$




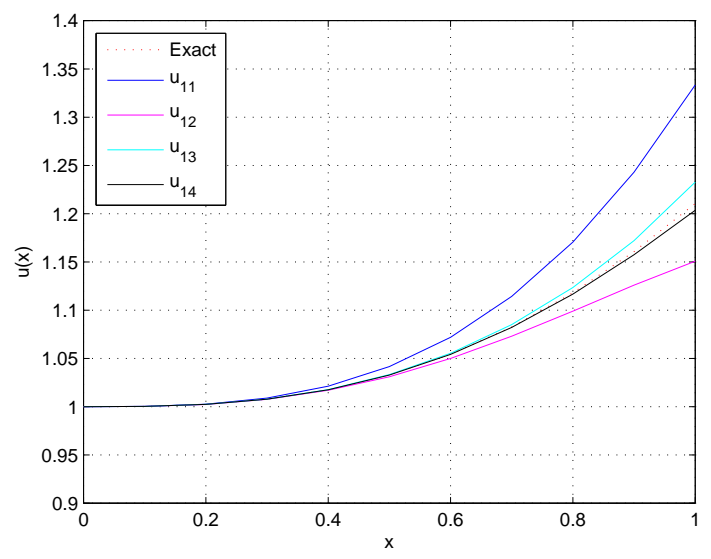

Fig. 2. Comparison of solution obtained by with exact solution of Example 2

Table 2. Percentage error of Example 2 with $P I A(1,1)$

\begin{tabular}{ccccc}
\hline & $\begin{array}{c}\text { \%Errorin } \\
u_{11}\end{array}$ & $\begin{array}{c}\text { \%Errorin } \\
u_{12}\end{array}$ & $\begin{array}{c}\text { \%Errorin } \\
u_{13}\end{array}$ & $\begin{array}{c}\text { \% Error in } \\
u_{14}\end{array}$ \\
\hline 0.10 & 0.0333330 & 0.0016660 & 0.0000670 & 0.0000020 \\
0.20 & 0.0531630 & 0.0266160 & 0.0021330 & 0.0001420 \\
0.30 & 0.6564110 & 0.1341400 & 0.0161870 & 0.0016230 \\
0.40 & 1.3438650 & 0.4203010 & 0.0681970 & 0.0091470 \\
0.50 & 2.3600120 & 1.0119050 & 0.2082600 & 0.0350570 \\
0.60 & 3.7794300 & 2.0563750 & 0.5195790 & 0.1054030 \\
0.70 & 5.6771020 & 3.7083950 & 1.1297560 & 0.2682540 \\
0.80 & 8.1217540 & 6.1157870 & 2.2272220 & 0.6046150 \\
0.90 & 11.1717300 & 9.4080460 & 4.0878070 & 1.2420120 \\
1.00 & 14.8712290 & 13.6904760 & 7.1204490 & 2.3697830 \\
\hline$\%$ & & & & \\
Mean & 4.8068030 & 3.6573707 & 1.5379660 & 0.4636040 \\
Error & & & & \\
\hline
\end{tabular}

subject to an initial condition $u(0)=0$. The exact solution of this problem is

$$
u(x)=\tan x
$$

When we apply Perturbation Iteration Algorithm , Eq. 5 implies

$$
\dot{u}^{c}=\frac{-\dot{u}+1}{\epsilon}+u^{2}
$$

On calculating the relevant terms and setting $\epsilon=1$. An initial trial function $u_{\circ}=x$ which satisfies initial condition is selected and using algorithm of perturbation Iteration Method, the approximate solution at each step are

$$
\begin{gathered}
u_{11}=x+\frac{1}{3} x^{3} \\
u_{12}=x+\frac{1}{3} x^{3}+\frac{2}{15} x^{5}+\frac{1}{63} x^{7}
\end{gathered}
$$

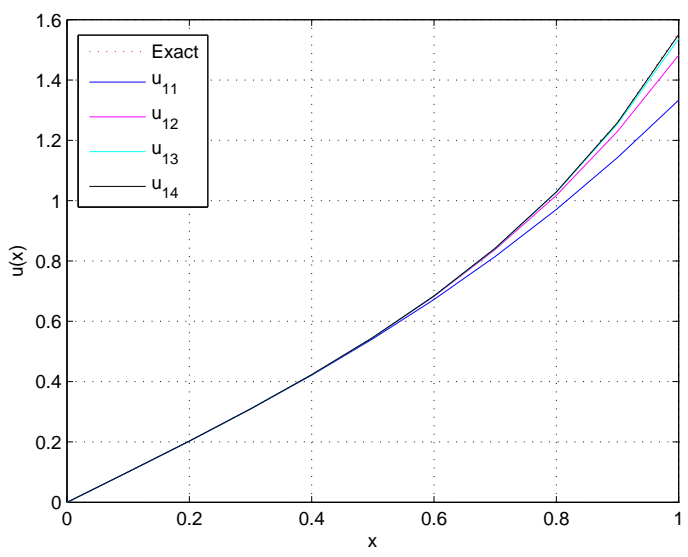

Fig. 3. Comparison of solution obtained by $P I A(1,1)$ with exact solution of Example 3

Table 3. Percentage error of Example 3 with $P I A(1,1)$

\begin{tabular}{ccccc}
\hline & $\begin{array}{c}\text { \%Errorin } \\
u_{11}\end{array}$ & $\begin{array}{c}\text { \%Errorin } \\
u_{12}\end{array}$ & $\begin{array}{c}\text { \%Error in } \\
u_{13}\end{array}$ & $\begin{array}{c}\text { \% Error in } \\
u_{14}\end{array}$ \\
\hline 0.1 & 0.001334 & $3.82 \times 10^{-6}$ & $8.5 \times 10^{-9}$ & $1.55 \times 10^{-11}$ \\
0.2 & 0.021395 & 0.000246 & $2.2 \times 10^{-6}$ & $1.64 \times 10^{-8}$ \\
0.3 & 0.108700 & 0.002838 & $5.76 \times 10^{-5}$ & $9.58 \times 10^{-7}$ \\
0.4 & 0.345295 & 0.016213 & 0.000593 & $1.77 \times 10^{-5}$ \\
0.5 & 0.848582 & 0.063179 & 0.00367 & 0.000174 \\
0.6 & 1.774032 & 0.193597 & 0.016543 & 0.001157 \\
0.7 & 3.318940 & 0.503213 & 0.060085 & 0.005873 \\
0.8 & 5.727436 & 1.160836 & 0.186888 & 0.024668 \\
0.9 & 9.297104 & 2.446852 & 0.517804 & 0.090133 \\
1.0 & 14.38765 & 4.807222 & 1.312974 & 0.296787 \\
\hline$\%$ & & & \\
Mean & 3.583047 & 0.91942 & 0.209862 & 0.041881 \\
Error & & & \\
\hline
\end{tabular}

$$
\begin{aligned}
u_{13}= & x+\frac{1}{3} x^{3}+\frac{2}{15} x^{5}+\frac{17}{315} x^{7}+\frac{38}{2835} x^{9}+\frac{134}{51975} x^{11}+ \\
& \frac{4}{12285} x^{13}+\frac{1}{59535} x^{15}
\end{aligned}
$$

$$
\begin{aligned}
u_{14}= & x+\frac{1}{3} x^{3}+\frac{2}{15} x^{5}+\frac{17}{315} x^{7}+\frac{62}{2835} x^{9}+\frac{1142}{155925} x^{11}+ \\
& \frac{13324}{6081075} x^{13}+\frac{377017}{638512875} x^{15}+\frac{1522814}{10854718875} x^{17}+ \\
& \frac{24022}{820945125} x^{19}+\frac{29756}{5746615875} x^{21}+\frac{12676238}{16962094524375} x^{23}+ \\
& \frac{256948}{3016973334375} x^{25}+\frac{100732}{14119435204875} x^{27}+ \\
& \frac{8}{21210236775} x^{29}+\frac{1}{109876902975} x^{31}
\end{aligned}
$$

which is very close to exact solution. 


\section{CONCLUSION}

The current publication puts forward a new method that proves effective in the determination of the approximate solution for the non-linear Riccati differential equation. To make our point more impressive, three examples from literature have been presented to determine that this method is the most convenient and simple one to use and its yields are more accurate that we could hope for. In fact, compared to older methods, it is better by many degrees. For the minor problem of the $C P U$ time for calculating $u_{11}, u_{12}, u_{13}$ and $u_{14}$ not being very high, all these calculation can easily be done by software Mathematica 9.0. Unlike the Adomian Decompostion Method, the Perturbation Iteration Algorithm PIA is independent of Adomian polynomials. While using this technique we have no requirement of the Lagrange multiplier, stationary conditions, calculating integrals, or correction functional. This eliminates the difficulties that occur in the Variational Iteration Method. Contrary to the Homotopy Perturbation Method, this method does not require solution of the functional equation in each iteration. Additionally, it entails a reduced amount of work when put in comparison with the Taylor Matrix technique. The solution that the method proposes for problems gives it an edge over other theories.

\section{CONFLICT OF INTERESTS}

The authors declare that there is no conflict of interests regarding the publication of this paper.

\section{ACKNOWLEDGMENT}

We thank the reviewers for their thorough efforts in editing our paper and highly appreciate the comments and constructive criticism that significantly contributed in improving the quality of the publication. The authors also thank Ms.Wishaal Khalid for proofreading our research paper.

\section{REFERENCES}

[1] Reid, W.T. (1972) Riccati Differential Equations. Academic Press, New York.

[2] Carinena, J.F, Marmo, G., Perelomov, A.M. \& Ranada M.F. (1998) Related operators and exact solutions of Schrodinger equations. International Journal of Modern Physics, 13. pp 4913-4929

[3] Scott, M.R. (1973) Invariant Imbedding and its Applications to Ordinary Differential Equations. Addison-Wesley, Boston.

[4] Tan, Y. \& Abbasbandy, S. (2008) Homotopy analysis method for quadratic Riccati differential equation. Commun. Nonlinear Sci. Numer. Simul., 13. pp 539-546

[5] Abbasbandy, S.(2007) A new application of Hes variational iteration method for quardratic Riccati differential equation by using Adomians polynomials. Journal of Computer Application, 207. pp 59-63

[6] El-Tawil, M.A., Bahnasawi, A.A. \& Abdel-Naby A. (2004) Solving Riccati differential equation using Adomians decomposition method. Applied Mathematics and Computation, 157. pp 503-514

[7] Abbasbandy, S. (2006) Iterated Hes homotopy perturbation method for quadratic Riccati differential equation. Applied Mathematics and Computation, 175. pp 581-589
[8] Abbasbandy, S. (2006) Homotopy Perturbation method for quadratic Riccati differential equation and comparision with Adomains Decomposition method. Applied Mathematics and Computation, 172. pp 485-90

[9] Geng,F., Lin, Y. \& Cui, M. (2009) A piecewise variational iteration method for Riccati differential equations. Comput. Math. Appl., 58. pp 2518-2522

[10] Gulsu, M. \& Sezer, M. (2006) On the solution of the Riccati equation by the Taylor matrix method. Applied Mathematics and Computation, 176. pp 414-421

[11] Akyuz-Dacoglu, A. \& Sezer, M. (2003) Chebyshev polynomial solutions of systems of high-order linear differential equations with variable coefficients. Applied Mathematics and Computation, 144. pp 237-247

[12] Nayfeh, A.H. (1973) it Perturbation Methods. WileyInterscience, New York.

[13] Skorokhod, A.V., Hoppensteadt, F.C. \& Salehi, H. (2002) Random Perturbation Methods with Applications in Science and Engineering. Springer, New York.

[14] Pakdemirli, M., Aksoy, Y. \& Boyaci, H. (2011) A new perturbation-iteration approach for first order differential equations. Mathematical and Computational Applications, 16(4). pp 890-899

[15] Aksoy, Y. \& Pakdemirli, M. (2010) New perturbationiteration solutions for Bratu-type equations. Computers and Mathematics with Applications, 59(8). pp -2808

[16] Aksoy, Y. Pakdemirli, M. \& Abbasbandy, S. (2012) New perturbation-iteration solutions for nonlinear heat transfer equations. International Journal of Numerical Methods for Heat and Fluid Flow, 22(7). pp 814-28 